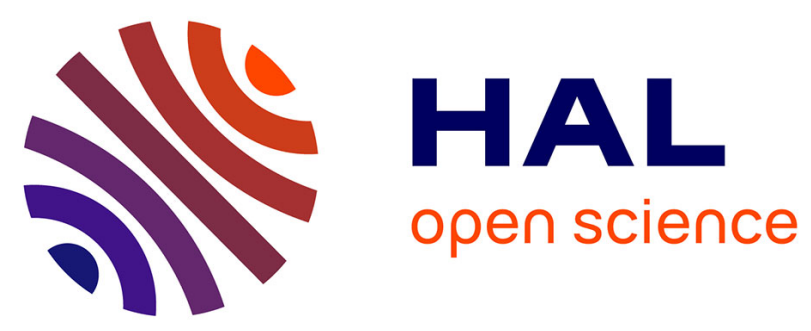

\title{
Interference Alignment with Delayed Differential Feedback for Time-Correlated MIMO Channels
}

Mingxin Zhou, Leiming Zhang, Lingyang Song, Mérouane Debbah, Bingli Jiao

\section{To cite this version:}

Mingxin Zhou, Leiming Zhang, Lingyang Song, Mérouane Debbah, Bingli Jiao. Interference Alignment with Delayed Differential Feedback for Time-Correlated MIMO Channels. IEEE ICC 2012, Aug 2012, Ottawa, Canada. pp.3741 - 3745, 10.1109/ICC.2012.6364535 . hal-00771205

\section{HAL Id: hal-00771205 \\ https://hal-centralesupelec.archives-ouvertes.fr/hal-00771205}

Submitted on 8 Jan 2013

HAL is a multi-disciplinary open access archive for the deposit and dissemination of scientific research documents, whether they are published or not. The documents may come from teaching and research institutions in France or abroad, or from public or private research centers.
L'archive ouverte pluridisciplinaire HAL, est destinée au dépôt et à la diffusion de documents scientifiques de niveau recherche, publiés ou non, émanant des établissements d'enseignement et de recherche français ou étrangers, des laboratoires publics ou privés. 


\title{
Interference Alignment with Delayed Differential Feedback for Time-Correlated MIMO Channels
}

\author{
Mingxin Zhou*, Leiming Zhang*, Lingyang Song*, Merouane Debbah ${ }^{\dagger}$, and Bingli Jiao* \\ ${ }^{*}$ The State Key Laboratory of Advanced Optical Communication Systems and Networks, \\ School of Electronics Engineering and Computer Science, Peking University, Beijing, China. 100871 \\ ${ }^{\dagger}$ SUPELEC, Alcatel-Lucent Chair in Flexible Radio, 3 rue Joliot-Curie, FR-91192 Gif Sur Yvette, France
}

\begin{abstract}
Interference alignment (IA) has been well recognized as an efficient approach to reduce interference at high signal to noise ratio (SNR). However, it demands global channel state information (CSI) at both transmitters and receivers for precoder design in order to maximize the multiplexing gain. In this paper, we discuss IA with delayed differential CSI feedback for time-correlated multiple input multiple output (MIMO) block fading channels. We consider the impact of distortion caused by channel estimation errors and quantized CSI feedback delay, and thus, find an optimal feedback interval to minimize this distortion, as well as the sum rate performance. Specifically, we derive the minimum differential feedback rate. And with the feedback-channel capacity constraint, we further study the relationship between the average sum rate and the feedback interval. Analytical results are verified by simulations.
\end{abstract}

\section{INTRODUCTION}

Interference alignment (IA) has been developed as an efficient technique to handle the interferences and then maximize the multiplexing gain or degree of freedom (Dof) in $K$-user interference channels [1]. It has been proved in [1] that with $K$ users single input single output (SISO) system, the capacity per user is given by

$$
C(S N R)=\frac{1}{2} \log (S N R)+o(\log (S N R)) .
$$

At high SNR, the $o(\log (S N R))$ term becomes negligible compared to $\log (S N R)$ and then every user is able to achieve a half Dof leading to a total of $\frac{K}{2}$ Dof. It indicates in [1] that, for the $K$-user MIMO case, the achievable Dof is $\min \left\{N_{t}, N_{r}\right\} \frac{K}{2}$, with $N_{t}$ and $N_{r}$ antennas at each transmitter and receiver, respectively.

Various approaches to design the precoder for IA schemes have been proposed in [1], [2] with closed-form solutions, and [3]-[5] with iterative solutions. All these aforementioned works depend on the assumption that each transmitter and receiver knows the full CSI. However, this is hard to realize as the capacity feedback channel is typically limited. It has been shown in [6]-[9] with limited feedback bits the maximum multiplexing gain can be also achievable in a SISO system as well as single input multiple output (SIMO) and MIMO system, where feedback rate is still high for practical IA implementation. However, when the channel is time varying, it would be sufficient to feedback the difference of consecutive CSI.

In this paper, we investigate IA scheme using differential feedback over time-correlated MIMO interference channels with distortion caused by the channel estimation errors and the quantized CSI feedback delay. The main contribution of this paper is summarized as follows. 1) We derive the minimum differential feedback rate for the time-correlated MIMO blockfading channels. 2) We investigate the relationship between the CSI distortion and feedback interval with feedback capacity constraint. Further, we prove the existence of optimal feedback interval in order to minimize the distortion. 3) The sum rate in our situation is provided as a function of the feedback interval, and we obtain an optimal value of average sum rate.

The rest of the paper is organized as follows. In section II, we describe the system model. Section III derives the relationship between the CSI distortion and the minimum differential feedback, as well as the existence of the optimal feedback interval for the time-correlated channels. In section IV, we discuss the sum rate performance based on this differential feedback IA scheme with distortion and the optimal feedback interval. In section $\mathrm{V}$, we provide the simulation results.

\section{System MOdEL}

In this paper, we consider a $K$-user IA scheme with time-correlated MIMO block-fading channels, as shown in Fig. 1, where each transmitter and receiver is equipped with $N_{t}$ and $N_{r}$ antennas respectively. The $i$-th transmitter $\mathcal{T}_{i}, i=1,2, \ldots, K$, transmits $d_{i}$ independent spatial data streams to its corresponding receiver $\mathcal{R}_{i}, i=1,2, \ldots, K$. The down-link channel coefficient $\mathbf{H}_{i k}(n), i, k=1,2, \cdots, K$, is constant throughout a block, and temporal correlated with each other in different block index $n$. The up-link channel is modeled as a limited feedback channel with a capacity constraint per fading block.

The received signal at the $i$-th receiver is defined as

$$
\begin{array}{r}
\mathbf{y}_{i}(n)=\mathbf{H}_{i i}(n) \mathbf{X}_{i}(n)+\sum_{k \neq i} \mathbf{H}_{i k}(n) \mathbf{X}_{k}(n)+\mathbf{n}_{i}(n), \\
i=1,2, \cdots, K
\end{array}
$$

where $\mathbf{y}_{i}$ denotes the $N_{r} \times 1$ output signal vector at $\mathcal{R}_{i}$, and $\mathbf{X}_{i}$ is the $N_{t} \times d_{i}$ input signal vector at $\mathcal{T}_{i} . \mathbf{H}_{i k}(n)$ represents the $n$-th channel coefficients from $\mathcal{T}_{k}$ to $\mathcal{R}_{i}$ which is a $N_{r} \times$ $N_{t}$ matrix with independent and identically distributed (i.i.d) complex Gaussian entries satisfying $\mathcal{C N}\left(0, \sigma_{h}^{2}\right) . \mathbf{n}_{i}$ is a $N_{r} \times$ 1 noise vector at the $i$-th receiver, modeled as i.i.d complex Gaussian variables satisfying $\mathcal{C N}\left(0, \sigma_{0}^{2}\right)$.

\section{A. Differential Feedback Model}

Using the maximum likelihood (ML) channel estimation, the CSI of transmitter $\mathcal{T}_{k}$ to receiver $\mathcal{R}_{i}$ can be estimated at 


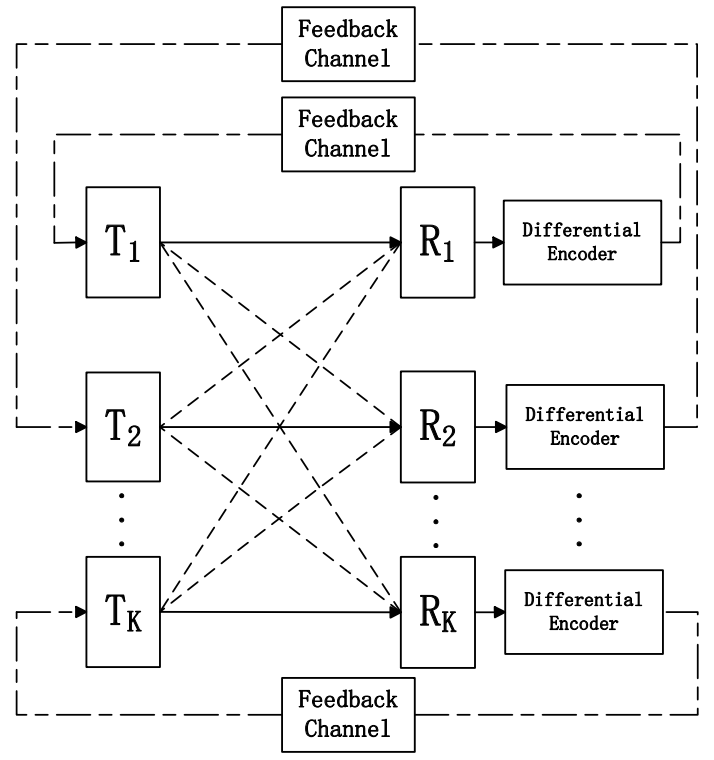

Fig. 1. System model: $K$-user IA with differential feedback

the $i$-th receiver with orthogonal pilots, and the estimation matrix can expressed as [10]

$$
\hat{\mathbf{H}}_{i k}(n)=\mathbf{H}_{i k}(n)+\mathbf{H}_{e}(n),
$$

where $\mathbf{H}_{i k}(n)$ is the actual channel coefficients, $\hat{\mathbf{H}}_{i k}(n)$ denotes the channel estimation matrix with ML estimation, and the entries are i.i.d. complex Gaussian variables $\mathcal{C N}\left(0, \sigma_{\hat{h}}^{2}\right)$. $\mathbf{H}_{e}(n)$ is the channel estimation error matrix, which is independent of $\mathbf{H}_{i k}(n)$, and the entries are independent complex Gaussian distributed with $\mathcal{C N}\left(0, \sigma_{\hat{h}}^{2}-\sigma_{h}^{2}\right)$.

As $\mathbf{H}_{e}(n)$ is independent of $\mathbf{H}_{i k}(n)$ in (3), $\mathbf{H}_{i k}(n)$ is written as [11]

$$
\mathbf{H}_{i k}(n)=\frac{\sigma_{h}^{2}}{\sigma_{\hat{h}}^{2}} \hat{\mathbf{H}}_{i k}(n)+\boldsymbol{\Psi}_{i k}(n),
$$

where $\sigma_{h}^{2}$ and $\sigma_{\hat{h}}^{2}$ denote the variances of $\mathbf{H}_{i k}(n)$ and $\hat{\mathbf{H}}_{i k}(n)$ respectively. $\boldsymbol{\Psi}_{i k}(n)$ is independent of $\hat{\mathbf{H}}_{i k}(n)$ with the entries are $\mathcal{C N}\left(0, \sigma_{h}^{2}\left(\sigma_{\hat{h}}^{2}-\sigma_{h}^{2}\right) / \sigma_{\hat{h}}^{2}\right)$.

The output of the feedback channel is quantized as [10]

$$
\hat{\mathbf{H}}_{i k}(n)=\overline{\mathbf{H}}_{i k}(n)+\mathbf{E}_{i k}(n),
$$

where $\overline{\mathbf{H}}_{i k}(n)$ denotes the channel quantization matrix with i.i.d. complex Gaussian entries, and $\mathbf{E}_{i k}(n)$ is an independent additive quantization error matrix, the entries are modeled as i.i.d. complex Gaussian variables satisfying $\mathcal{C N}\left(0, \delta_{d}\right)$, and $\delta_{d}$ represents the average channel quantization distortion.

For time-correlated channel, we consider the differential feedback. As the previous channel quantization matrix $\overline{\mathbf{H}}_{i k}(n)$ is known both at receiver and transmitter, the differential CSI can be formulated as

$$
\mathbf{H}_{d, i k}(n)=\operatorname{Diff}\left(\hat{\mathbf{H}}_{i k}(n) \mid \overline{\mathbf{H}}_{i k}(n-1)\right),
$$

where $\mathbf{H}_{d, i k}(n)$ represents the differential CSI between $\hat{\mathbf{H}}_{i k}(n)$ and $\overline{\mathbf{H}}_{i k}(n-1)$, and $\operatorname{Diff}(\cdot)$ denotes the differential function. Furthermore, as we assumed that the CSI feedback channel has a limited feedback bits $b$ per fading block with T blocks in each feedback interval, the total number of bits to quantize the CSI is $T \times b$.

\section{B. CSI Delay Model}

The time-correlated channel can be modeled as a first-order Autoregressive process (AR1) [12], where the channel remains constant for a block duration and changes from block to block. The current channel fading matrix $\mathbf{H}_{i k}(n)$ and its delayed version $\mathbf{H}_{i k}(n-1)$ can be expressed as

$$
\mathbf{H}_{i k}(n)=\alpha \mathbf{H}_{i k}(n-1)+\sqrt{1-\alpha^{2}} \mathbf{W}_{i k}(n),
$$

where $\mathbf{W}_{i k}(n)$ is a noise matrix, which is independent of $\mathbf{H}_{i k}(n-1)$, and the entries are i.i.d. complex Gaussian variables $\mathcal{C N}\left(0, \sigma_{h}^{2}\right)$. The parameter $\alpha$ is the time autocorrelation coefficient, which is given by the zero-order Bessel function of first kind $\alpha=J_{0}\left(2 \pi f_{d} \tau\right)$, where $f_{d}$ denotes the maximum Doppler frequency in Hertz, and $\tau$ denotes the time interval. In the block fading feedback system, given the number of the feedback block $T$, the time interval can be calculated as $\tau=T \cdot \tau_{\text {block }}$, where $\tau_{\text {block }}$ is the duration of every block.

Considering $n=N T+t$, the channel coefficient of the $t$-th block in the $N$-th period $\overline{\mathbf{H}}_{i k}(n)$ is expressed as $\overline{\mathbf{H}}_{i k}(N, t)$. In a practical communication system, $\overline{\mathbf{H}}_{i k}(N, 1)$ can be only used in the next feedback interval $\left\{\mathbf{H}_{i k}(N+1,1), \mathbf{H}_{i k}(N+1,2), \ldots, \mathbf{H}_{i k}(N+1, T)\right\}$. Hence, the feedback delay is $T$.

Next, we derive the relationship between $\mathbf{H}_{i k}(N+1, t)$ and $\overline{\mathbf{H}}_{i k}(N, 1)$. From (7), we obtain

$$
\begin{aligned}
\mathbf{H}_{i k}(N+1, t)=\alpha_{t}\left(\alpha_{T} \mathbf{H}_{i k}(N, 1)+\right. & \left.\sqrt{1-\alpha_{T}^{2}} \mathbf{W}_{T}\right) \\
& +\sqrt{1-\alpha_{t}^{2}} \mathbf{W}_{t}
\end{aligned}
$$

where $\alpha_{t}=J_{0}\left(2 \pi f_{d} t \tau_{\text {block }}\right)$ denotes the time autocorrelation coefficient of $t$ blocks, and $\alpha_{T}=J_{0}\left(2 \pi f_{d} T \tau_{\text {block }}\right)$ denotes the time autocorrelation between adjacent feedback periods.

Substituting (4), (5) into (8) yields

$$
\begin{aligned}
& \mathbf{H}_{i k}(N+1, t)=\alpha_{t} \alpha_{T} \frac{\sigma_{h}^{2}}{\sigma_{\hat{h}}^{2}} \overline{\mathbf{H}}_{i k}(N, 1)+\alpha_{t} \alpha_{T} \frac{\sigma_{h}^{2}}{\sigma_{\hat{h}}^{2}} \mathbf{H}_{e}(N, 1) \\
& +\alpha_{t} \alpha_{T} \boldsymbol{\Psi}_{i k}(N, 1)+\alpha_{t} \sqrt{1-\alpha_{T}^{2}} \mathbf{W}_{T}+\sqrt{1-\alpha_{t}^{2}} \mathbf{W}_{t} .
\end{aligned}
$$

From (9), we can see that the first term is correlated with the quantized CSI and other terms act as the distortion of the feedback CSI.

\section{Relationship BetWeEn the Distortion AND THE FEEDBACK INTERVAL}

In this section, we discuss the CSI distortion with channel estimation and delayed CSI feedback. First, we derive the minimum differential feedback rate of time-correlated MIMO block-fading channels, assuming that a CSI distortion is given. When the previous channel quantization matrix $\overline{\mathbf{H}}_{i k}(n-1)$ is known at both the receivers and the transmitters, the minimum differential feedback rate can be written as

$$
\begin{array}{r}
N_{f}=\inf \left\{I\left(\hat{\mathbf{H}}_{i k}(n) ; \overline{\mathbf{H}}_{i k}(n) \mid \overline{\mathbf{H}}_{i k}(n-1)\right):\right. \\
\left.\mathbb{E}\left[d\left(\hat{\mathbf{H}}_{i k}(n), \overline{\mathbf{H}}_{i k}(n)\right)\right] \leq \Delta_{d}\right\},
\end{array}
$$

where $\inf \{\cdot\}$ denotes an infimum function, $I\left(\hat{\mathbf{H}}_{i k}(n) ; \overline{\mathbf{H}}_{i k}(n) \mid \overline{\mathbf{H}}_{i k}(n-1)\right) \quad$ represents the mutual information between $\hat{\mathbf{H}}_{i k}(n)$ and $\overline{\mathbf{H}}_{i k}(n)$ when $\overline{\mathbf{H}}_{i k}(n-1)$ is given. $d\left(\hat{\mathbf{H}}_{i k}(n), \overline{\mathbf{H}}_{i k}(n)\right)=\left\|\hat{\mathbf{H}}_{i k}(n)-\overline{\mathbf{H}}_{i k}(n)\right\|^{2}$ denotes the quantization distortion. 
Since the entries of $\mathbf{H}_{i k}, \hat{\mathbf{H}}_{i k}$ and $\overline{\mathbf{H}}_{i k}$ are i.i.d. complex Gaussian variables, the minimum differential feedback rate is written as

$$
\begin{array}{r}
N_{f}=\inf \left\{N_{r} N_{t} \cdot I\left(\hat{h}_{i k}(n) ; \bar{h}_{i k}(n) \mid \bar{h}_{i k}(n-1)\right):\right. \\
\left.\mathbb{E}\left[d\left(\hat{h}_{i k}(n), \bar{h}_{i k}(n)\right)\right] \leq \delta_{d}\right\},
\end{array}
$$

where $\delta_{d}=\Delta_{d} /\left(N_{r} N_{t}\right)$ denotes the average channel quantization distortion, $\hat{h}_{i k}(n), \bar{h}_{i k}(n)$ and $\bar{h}_{i k}(n-1)$ represent the entries of $\hat{\mathbf{H}}_{i k}(n), \overline{\mathbf{H}}_{i k}(n)$ and $\overline{\mathbf{H}}_{i k}(n-1)$, respectively.

Lemma 1: Given a one-dimensional channel quantization distortion constraint $\delta_{d}$, and the $(n-1)$-th channel quantization element $\bar{h}_{i k}(n-1)$, the mutual information $I\left(\hat{h}_{i k}(n) ; \bar{h}_{i k}(n) \mid \bar{h}_{i k}(n-1)\right)$ is calculated as

$I \geq \log \left(\alpha^{2}\left(\frac{\sigma_{h}^{2}}{\sigma_{\hat{h}}^{2}}\right)^{2}+\frac{\left(1-\alpha^{2}\right)}{\delta_{d}} \sigma_{h}^{2}+\frac{\left(\sigma_{\hat{h}}^{2}-\sigma_{h}^{2}\right)}{\delta_{d}}\left(1+\alpha^{2} \frac{\sigma_{h}^{2}}{\sigma_{\hat{h}}^{2}}\right)\right)$.

where $\sigma_{h}^{2}, \sigma_{\hat{h}}^{2}$ denote the variances of $h$ and $\hat{h}$ respectively, and $\alpha$ is the time autocorrelation coefficient.

The proof of Lemma 1 can be found in Appendix A. Substituting (12) into (11), the minimum feedback rate in the differential feedback system is given by

$$
\begin{aligned}
N_{f}=N_{r} N_{t} \log & \left(\alpha^{2}\left(\frac{\sigma_{h}^{2}}{\sigma_{\hat{h}}^{2}}\right)^{2}+\frac{\left(1-\alpha^{2}\right)}{\delta_{d}} \sigma_{h}^{2}\right. \\
+ & \left.\frac{\left(\sigma_{\hat{h}}^{2}-\sigma_{h}^{2}\right)}{\delta_{d}}\left(1+\alpha^{2} \frac{\sigma_{h}^{2}}{\sigma_{\hat{h}}^{2}}\right)\right) .
\end{aligned}
$$

From (13), we can see the relationship between the minimum differential feedback rate and the average CSI distortion $\delta_{d}$. Thus $\delta_{d}$ can be written as

$$
\delta_{d}=\frac{\sigma_{\hat{h}}^{2}-\left(\frac{\sigma_{h}^{2}}{\sigma_{\hat{h}}^{2}}\right)^{2} \sigma_{\hat{h}}^{2} \cdot \alpha^{2}}{2^{\frac{N_{f}}{N_{r} N_{t}}}-\alpha^{2}\left(\frac{\sigma_{h}^{2}}{\sigma_{\hat{h}}^{2}}\right)^{2}} .
$$

In the block fading system, the number of feedback block and feedback delay is $T$, which means that $\alpha$ in (14) is given by $\alpha_{T}=J_{0}\left(2 \pi f_{d} T \tau_{b l o c k}\right)$ and $N_{f}=T b$. Then, combining (22) and (14), with causal feedback constraint, the quantization distortion $d$ between $\hat{h}_{i k}(n)$ and $\bar{h}_{i k}(n-1)$ is given by

$$
\begin{aligned}
d= & \alpha_{T}^{2}\left(\frac{\sigma_{h}^{2}}{\sigma_{\hat{h}}^{2}}\right)^{2} \frac{\sigma_{\hat{h}}^{2}-\left(\frac{\sigma_{h}^{2}}{\sigma_{\hat{h}}^{2}}\right)^{2} \sigma_{\hat{h}}^{2} \cdot \alpha_{T}^{2}}{2^{\frac{T b}{N_{r} N_{t}}}-\alpha_{T}^{2}\left(\frac{\sigma_{h}^{2}}{\sigma_{\hat{h}}^{2}}\right)^{2}}+\alpha_{T}^{2} \frac{\sigma_{h}^{2}\left(\sigma_{\hat{h}}^{2}-\sigma_{h}^{2}\right)}{\sigma_{\hat{h}}^{2}} \\
& +\left(1-\alpha_{T}^{2}\right) \sigma_{h}^{2}+\left(\sigma_{\hat{h}}^{2}-\sigma_{h}^{2}\right) \\
= & \alpha_{T}^{2} \frac{\sigma_{h}^{4}}{\sigma_{\hat{h}}^{2}}\left(\frac{1-2^{\frac{T b}{N_{r} N_{t}}}}{2^{\frac{T b}{N_{r} N_{t}}}-\alpha_{T}^{2}\left(\frac{\sigma_{h}^{2}}{\sigma_{\hat{h}}^{2}}\right)^{2}}\right)+\sigma_{\hat{h}}^{2}
\end{aligned}
$$

which is a measurement of the quality of the periodic feedback system. From (15), we can obtain that $d$ is a function of $\alpha_{T}$ and $T$. In a periodic feedback system with limited feedback, $\alpha_{T}$ is related to $T$. Therefore, the quality of feedback information measured by $d(T)$ can be expressed as a function of $T$.

As we can see in (15), with extension of feedback period, in one hand, the increase of the feedback rate results in the decrease of quantization error. But in the other hand, the time correlation reduces and feedback delay becomes worse. Intuitively, there exists an optimal $T$ to minimize the distortion
$d(T)$. The detailed proof is given in Appendix B. Moreover, as the loss of sum rate is mainly caused by the distortion in (15), the sum rate is negatively correlated with distortion.

\section{INTERFERENCE AlignMENT WITH PERIODIC DIFFERENTIAL FEEDBACK}

In this section, we consider IA with periodic differential feedback, as well as the impact of CSI distortion caused by imperfect estimation quantization and delay on average sum rate in one feedback period.

Each source transmits a linear combination of $d_{i}$ scalar symbols $\mathbf{T}_{i}=\left\{t_{i}^{1}, t_{i}^{2}, \cdots, t_{i}^{d i}\right\}$ to the corresponding destination by modulating the symbols onto the transmit direction vectors $\mathbf{V}_{i}=\left\{\mathbf{v}_{i}^{1}, \mathbf{v}_{i}^{2}, \cdots, \mathbf{v}_{i}^{d i}\right\}$, that is,

$$
\mathbf{X}_{i}=\mathbf{V}_{i} \mathbf{T}_{i}=\sum_{m=1}^{d_{i}} \mathbf{v}_{i}^{m} t_{i}^{m}
$$

where the direction vectors $v_{i}^{m}$ is a $N_{t} \times 1$ complex Gaussian vector with $\left\|\mathbf{v}_{i}^{m}\right\|^{2}=1$, and $E\left\|t_{i}^{m}\right\|^{2}=\frac{P}{d_{i}}$ to satisfy the power constraint.

When all of the interference is aligned to an interference subspace of the receiver space, each receiver computes a receive direction vector $\mathbf{U}_{i}=\left\{\mathbf{u}_{i}^{1}, \mathbf{u}_{i}^{2} \cdots, \mathbf{u}_{i}^{d_{i}}\right\}$ to zero-force the interference, where $\left\|\mathbf{u}_{i}^{m}\right\|^{2}=1$.

As each transmitter and receiver knows the previous quantized CSI $\overline{\mathbf{H}}_{i k}(N-1,1)$, each receiver needs to estimate $\mathbf{H}_{i k}(N, 1)$ to calculate the differential information and feeds them back to transmitter through feedback channels during $T$ blocks of the $N$-th feedback period. With casual constraint, quantization CSI $\overline{\mathbf{H}}_{i k}(N, 1)$ can be only recovered by the $k$-th transmitter at the $(N+1)$-th feedback period and use them to design the precoding vectors $\overline{\mathbf{V}}_{i}(N+1)$ and $\overline{\mathbf{U}}_{i}(N+1), i=1,2, \cdots, K$, respectively, satisfying the following conditions

$$
\begin{aligned}
& \left(\overline{\mathbf{u}}_{i}^{m}(N+1)\right)^{H} \overline{\mathbf{H}}_{i i}(N, 1) \overline{\mathbf{v}}_{i}^{p}(N+1)=0, \forall i, m \neq p, \\
& \left(\overline{\mathbf{u}}_{i}^{m}(N+1)\right)^{H} \overline{\mathbf{H}}_{i k}(N, 1) \overline{\mathbf{v}}_{k}^{p}(N+1)=0, \forall m, p, k \neq i, \\
& \left.\left|\left(\overline{\mathbf{u}}_{i}^{m}(N+1)\right)^{H} \overline{\mathbf{H}}_{i i}(N, 1) \overline{\mathbf{v}}_{i}^{m}(N+1)\right| \geq c, \forall i, m, \quad 17\right)
\end{aligned}
$$

where $c$ is a positive constant independent of $P$.

Finally, the received signal at the $i$-th receiver node is written as

$$
\begin{array}{r}
\left(\overline{\mathbf{u}}_{i}^{m}(N+1)\right)^{H} \mathbf{y}_{i}=\left(\overline{\mathbf{u}}_{i}^{m}(N+1)\right)^{H} \mathbf{H}_{i i}(N+1, t) \overline{\mathbf{v}}_{i}^{m}(N+1) t_{i}^{m} \\
+\sum_{p \neq m}^{d_{i}}\left(\overline{\mathbf{u}}_{i}^{m}(N+1)\right)^{H} \mathbf{H}_{i i}(N+1, t) \overline{\mathbf{v}}_{i}^{p}(N+1) t_{i}^{p} \\
+\sum_{k \neq i}^{K} \sum_{p=1}^{d_{i}}\left(\overline{\mathbf{u}}_{i}^{m}(N+1)\right)^{H} \mathbf{H}_{i k}(N+1, t) \overline{\mathbf{v}}_{k}^{p}(N+1) t_{k}^{p} \\
+\left(\overline{\mathbf{u}}_{i}^{m}(N+1)\right)^{H} n_{i},
\end{array}
$$

for $t=1,2, \cdots, T$.

For simplicity, we ignore the label $(N, t)$ in the flowing. Substituting (9) into (18), and considering the IA conditions in (17), it yields (19), where the second term and the third term are residual interference caused by imperfect CSI.

From (19), the achievable average sum rate is given by 


$$
\begin{array}{r}
\left(\overline{\mathbf{u}}_{i}^{m}\right)^{H} \mathbf{y}_{i}=\left(\overline{\mathbf{u}}_{i}^{m}\right)^{H} \mathbf{H}_{i i} \overline{\mathbf{v}}_{i}^{m} t_{i}^{m}+\sum_{p \neq m}^{d_{i}}\left(\overline{\mathbf{u}}_{i}^{m}\right)^{H}\left(\alpha _ { t } \left(\alpha_{T} \frac{\sigma_{h}^{2}}{\sigma_{\hat{h}}^{2}}\right.\right. \\
+\sum_{k \neq i}^{K} \sum_{p=1}^{d_{i}}\left(\overline{\mathbf{u}}_{i}^{m}\right)^{H}\left(\alpha _ { t } \left(\alpha_{T} \frac{\sigma_{h}^{2}}{\sigma_{\hat{h}}^{2}} \mathbf{H}_{e}+\alpha_{T} \mathbf{\Psi}_{i k}\right.\right. \\
\bar{R}_{\text {sum }}=\frac{1}{T} \sum_{t=1}^{T} \sum_{i=1}^{K} \sum_{m=1}^{d_{i}} \log \left(1+\frac{\left.\frac{P_{w}}{d_{i}}\left(\bar{u}_{i}^{m}\right)^{H} H_{i i} \bar{v}_{i}^{m}\right|^{2}}{\mathcal{I}_{i 1}(t)+\mathcal{I}_{i 2}(t)+\sigma_{0}^{2}}\right) .
\end{array}
$$

As we can see in (20), the average sum rate is also a function of $T$. With the analysis in section III, the average sum rate is a monotonic convex function of $T$, and this will be further verified in section $\mathrm{V}$ by numeric results.

\section{Simulation Results and Discussions}

In this section, we first provide the simulation results of the distortion caused by estimation quantization and delay in a feedback period. Then, we discuss the relationship between the average sum rate and feedback intervals. Due to the analysis in previous sections, they can both generate optimal values which are equal in the simulation results.

A. Distortion Caused by Channel Estimation and Delay

The relationship between feedback interval and distortion has been shown in Fig. 2. In this section, we consider the variances of the channel coefficient, noise and estimation error are $\sigma_{h}^{2}=1, \sigma_{0}^{2}=1, \sigma_{e}^{2}=\sigma_{\hat{h}}^{2}-\sigma_{h}^{2}=0.2$, respectively. Also, we assume the duration of each fading block is $\tau=1 \mathrm{~ms}$, and the Doppler frequency is $9.26 \mathrm{~Hz}$ corresponding to $5 \mathrm{~km} / \mathrm{h}$ moving speed and $2 \mathrm{GHz}$ Carrier Frequency.

As shown in Fig. 2, $d(T)$ is a monotonic convex function of $T$ in the limited and periodical feedback system. The distortion decrease at first is due to the increase of feedback rate caused by extension of feedback period. But when $T$ becomes large, the time correlation $\alpha_{T}$ decreases, which causes the distortion gradually increase. Additionally, we can also see as the limited constraint per fading block $b$ increases, the distortion decreases.

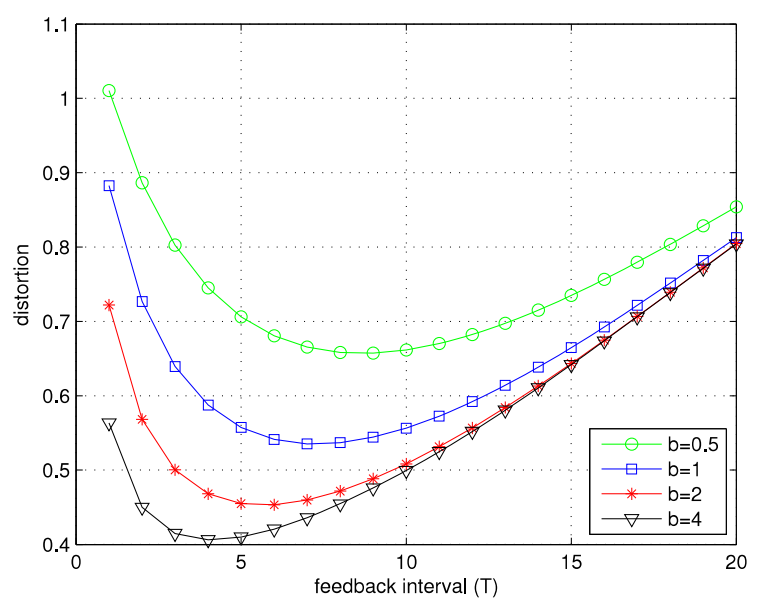

Fig. 2. The relationship between average distortion and feedback blocks, for $N_{r}=2, N_{t}=2, \sigma_{h}^{2}=1$ and $\sigma_{\hat{h}}^{2}=1.2$.

\section{B. Average Sum Rate}

We consider the similar system setup as section A. In addition, we assume there are 3 users in the interference channel where each node has 2 antennas with transmit power $P=15$ $\mathrm{dB}$ and $\left\{d_{i}=1, i=1,2,3\right\}$ data stream. The precoding and decoding vectors are calculated by the general interferenceminimization scheme [5]. We also give the numerical results in Fig. 3. Note that the relationship between $\bar{R}_{\text {sum }}$ and $T$ is consistent with the consequence of distortion as the analysis in section III, as well as the optimal values. Furthermore, as $b$ increases, the average sum rate improves as shown in Fig. 3.

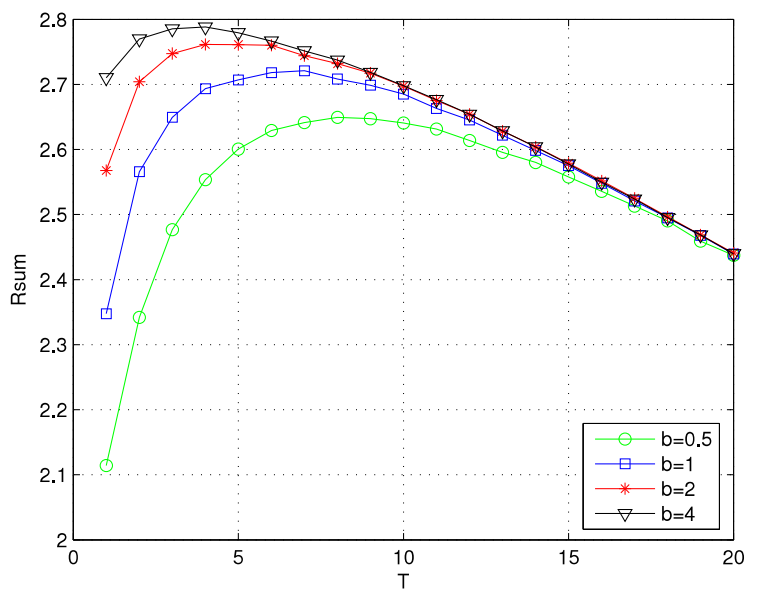

Fig. 3. The relationship between average sum rate and feedback blocks, for $K=3, N_{r}=2, N_{t}=2, \sigma_{h}^{2}=1, \sigma_{\hat{h}}^{2}=1.2, P=15 \mathrm{~dB}$ per user and $\left\{d_{i}=1, i=1,2,3\right\}$.

\section{Conclusions}

In this paper, we have investigated the delayed differential feedback IA scheme over time-correlated MIMO block fading channels. We found the CSI distortion was a monotonic convex function of the feedback interval, and we proved the existence of the optimal value of feedback interval to minimize the distortion. We also discussed the relationship between the average sum rate in one feedback period and the feedback interval, which is similar as CSI distortion. In the end, we provided the numeric results to support the analysis.

\section{ACKNOWLEDGEMENT}

This work was partially supported by the National Natural Science Foundation of China under Grant number 61061130561 and 60972009, and by National Science and Technology key project under grant Number 2011ZX03005002 and 2010ZX03003-003.

\section{APPENDIX A}

PROOF OF LEMMA 1

Combining (3), (4), (5) and (7), we get

$$
\begin{array}{r}
\hat{\mathbf{H}}_{i k}(n)=\alpha \frac{\sigma_{h}^{2}}{\sigma_{\hat{h}}^{2}} \overline{\mathbf{H}}_{i k}(n-1)+\alpha \frac{\sigma_{h}^{2}}{\sigma_{\hat{h}}^{2}} \mathbf{E}_{i k}(n-1)+\alpha \boldsymbol{\Psi}_{i k}(n-1) \\
+\sqrt{1-\alpha^{2}} \mathbf{W}_{i k}(n)+\mathbf{H}_{e}(n) .
\end{array}
$$


The one-dimensional form of (21) is expressed as

$$
\begin{array}{r}
\hat{h}_{i k}(n)=\alpha \frac{\sigma_{h}^{2}}{\sigma_{\hat{h}}^{2}} \bar{h}_{i k}(n-1)+\alpha \frac{\sigma_{h}^{2}}{\sigma_{\hat{h}}^{2}} e_{i k}(n-1)+\alpha \psi_{i k}(n-1) \\
+\sqrt{1-\alpha^{2}} w_{i k}(n)+h_{e}(n) .
\end{array}
$$

The mutual information $I\left(\hat{h}_{i k}(n) ; \bar{h}_{i k}(n) \mid \bar{h}_{i k}(n-1)\right)$ in (11) is written as

$I=h\left(\hat{h}_{i k}(n) \mid \bar{h}_{i k}(n-1)\right)-h\left(\hat{h}_{i k}(n) \mid \bar{h}_{i k}(n), \bar{h}_{i k}(n-1)\right)$ substituting (22) into (23) yields

$$
\begin{array}{r}
I=h\left(\alpha \frac{\sigma_{h}^{2}}{\sigma_{\hat{h}}^{2}} e_{i k}(n-1)+\alpha \psi_{i k}(n-1)+\sqrt{1-\alpha^{2}} w_{i k}(n)+h_{e}(n)\right) \\
-h\left(h_{e}(n) \mid \bar{h}_{i k}(n-1)\right) .
\end{array}
$$

Considering the inequality $h\left(e_{i k}(n) \mid \bar{h}_{i k}(n-1)\right)$ $h\left(e_{i k}(n)\right)$, and $h\left(e_{i k}(n)\right)=h\left(e_{i k}(n-1)\right)$, it yields

$$
\begin{array}{r}
I \geq h\left(\alpha \frac{\sigma_{h}^{2}}{\sigma_{\hat{h}}^{2}} e_{i k}(n-1)+\alpha \psi_{i k}(n-1)+\sqrt{1-\alpha^{2}} w_{i k}(n)+h_{e}(n)\right) \\
-h\left(e_{i k}(n-1)\right) .
\end{array}
$$

As $e_{i k}(n-1), \psi_{i k}(n-1), w_{i k}(n)$ and $h_{e}(n)$ are complex Gaussian variables and independent with each other, according to the rate distortion theory of continuous-amplitude sources, we have

$I \geq \log \left(\alpha^{2}\left(\frac{\sigma_{h}^{2}}{\sigma_{\hat{h}}^{2}}\right)^{2}+\frac{\left(1-\alpha^{2}\right)}{\delta_{d}} \sigma_{h}^{2}+\frac{\left(\sigma_{\hat{h}}^{2}-\sigma_{h}^{2}\right)}{\delta_{d}}\left(1+\alpha^{2} \frac{\sigma_{h}^{2}}{\sigma_{\hat{h}}^{2}}\right)\right)$

\section{APPENDIX B}

\section{Proof of Existence of THE OPTIMAL FeEdBACK} INTERVAL $(\mathrm{T})$

We prove the existence of the optimal $T$ by finding the extreme value of $d(T)$. For simplicity, we assume $x=$ $2 \pi f_{d} T \tau_{\text {block }}$ is a continuous variable. Thus, the time correlation is expressed as $\alpha_{T}=J_{0}\left(2 \pi f_{d} T \tau_{\text {block }}\right)=J_{0}(x)$, and feedback block is calculated as a nearest integer of $T=x /\left(2 \pi f_{d} \tau_{\text {block }}\right)$. Then, (15) is rewritten as

$$
d(x)=J_{0}(x)^{2}\left(\frac{\sigma_{h}^{4}}{\sigma_{\hat{h}}^{2}}\right)\left(\frac{1-2^{k x}}{2^{k x}-J_{0}(x)^{2}\left(\frac{\sigma_{h}^{2}}{\sigma_{\hat{h}}^{2}}\right)^{2}}\right)+\sigma_{\hat{h}}^{2},
$$

where $k=b /\left(2 \pi N_{r} N_{t} f_{d} \tau_{\text {block }}\right)$, and $d(x)$ is a continuously differential function of $x$. Then, the first-order derivative of $d(x)$ is given by

$$
\begin{aligned}
& \frac{d}{d x} d(x)=2^{k x} J_{0}(x)\left(\frac{\sigma_{h}^{4}}{\sigma_{\hat{h}}^{2}}\right)\left(\frac{2\left(2^{k x}-1\right) J_{1}(x)}{\left(2^{k x}-J_{0}(x)^{2}\left(\frac{\sigma_{h}^{2}}{\sigma_{\hat{h}}^{2}}\right)^{2}\right)^{2}}\right. \\
& \left.-\frac{k \ln 2 \cdot\left(J_{0}(x)-\left(\frac{\sigma_{h}^{2}}{\sigma_{h}^{2}}\right)^{2} J_{0}(x)^{3}\right)}{\left(2^{k x}-J_{0}(x)^{2}\left(\frac{\sigma_{h}^{2}}{\sigma_{\hat{h}}^{2}}\right)^{2}\right)^{2}}\right),
\end{aligned}
$$

where $J_{1}(x)=-\frac{d}{d x} J_{0}(x)$, and $J_{n}(x)$ denotes a first kind n-th order Bessel function. As $\lim _{x \rightarrow 0} J_{0}(x)=1$ and $\lim _{x \rightarrow 0} J_{1}(x)=0$, the first-order derivative of $d(x)$ is given by

$$
\left.\frac{d}{d x} d(x)\right|_{x \rightarrow 0}=-\frac{\left(\frac{\sigma_{h}^{4}}{\sigma_{\hat{h}}^{2}}\right) k \ln 2 \cdot\left(1-\left(\frac{\sigma_{h}^{2}}{\sigma_{\hat{h}}^{2}}\right)^{2}\right)}{\left(1-\left(\frac{\sigma_{h}^{2}}{\sigma_{\hat{h}}^{2}}\right)^{2}\right)^{2}}<0 .
$$

By taking a special value (there may exist many other values), $x=3 / 2$, which enables the value of the first order Bessel function larger than the zero-order one, i.e. $J_{1}\left(\frac{3}{2}\right)>J_{0}\left(\frac{3}{2}\right)$, we have

$$
\begin{aligned}
& \left.\frac{d}{d x} d(x)\right|_{x=\frac{3}{2}} \\
& >2^{\frac{3}{2} k} J_{0}\left(\frac{3}{2}\right)^{2}\left(\frac{\sigma_{h}^{4}}{\sigma_{\hat{h}}^{2}}\right) \frac{2\left(2^{\frac{3}{2} k}-1\right)-k \ln 2 \cdot\left(1-\left(\frac{\sigma_{h}^{2}}{\sigma_{\hat{h}}^{2}}\right)^{2} J_{0}\left(\frac{3}{2}\right)^{2}\right)}{\left(2^{\frac{3}{2} k}-J_{0}\left(\frac{3}{2}\right)^{2}\left(\frac{\sigma_{h}^{2}}{\sigma_{\hat{h}}^{2}}\right)^{2}\right)^{2}} \\
& >2^{\frac{3}{2} k} J_{0}\left(\frac{3}{2}\right)^{2}\left(\frac{\sigma_{h}^{4}}{\sigma_{\hat{h}}^{2}}\right) \frac{2\left(2^{\frac{3}{2} k}-1\right)-k \ln 2}{\left(2^{\frac{3}{2} k}-J_{0}\left(\frac{3}{2}\right)^{2}\left(\frac{\sigma_{h}^{2}}{\sigma_{\hat{h}}^{2}}\right)^{2}\right)^{2}} .
\end{aligned}
$$

Using Taylor expansion, we have $2^{\frac{3}{2} k}=1+\frac{3}{2} \ln 2 \cdot k+$ $\frac{1}{2 !}\left(\frac{3}{2} \ln 2 \cdot k\right)^{2}+\ldots$ Therefore, we obtain

$$
2\left(2^{\frac{3}{2} k}-1\right)-k \ln 2>3 k \ln 2-k \ln 2>0 .
$$

Substituting (31) into (30), it yields

$$
\left.\frac{d}{d x} d(x)\right|_{x=\frac{3}{2}}>0 \text {. }
$$

As $\frac{d}{d x} d(x)$ is a continuous function of $x$, combining (29) and (32), we can obtain there exists a $x$ satisfying $\frac{d}{d x} d(x)=0$, when $0<x<\frac{3}{2}$. Thus, the existence of the optimal $T$ is proved.

\section{REFERENCES}

[1] V. R. Cadambe and S. A. Jafar, "Interference alignment and degrees of freedom of the K-user interference channel," IEEE Transactions on Information Theory, vol. 54, no. 8, pp. 3425-3441, Aug. 2008.

[2] H. Sung, S. H. Park, K. J. Lee and I. Lee, "Linear precoder designs for $\mathrm{K}$-user interference channels" IEEE. Transactions on Wireless Communications, vol. 9, no. 1, 291-301, Jan. 2010.

[3] S. W. Peters and R. W. Heath, Jr., "Cooperative algorithms for MIMO interference channels" IEEE. Transactions on Vehicular Technology, vol. 60, no. 1, Jan. 2011

[4] S. W. Peters and R. W. Heath, Jr., "Interference alignment via alternating minization" in Proc. IEEE ICASSP., Taipei, China, Apr. 2009.

[5] K. Gomadam, V. R. Cadambe and S. A. Jafar, "Approaching the capacity of wireless networks through distributed interference alignment," in Proc. IEEE. GLOBECOM., New Orleans, LO., Dec. 2008.

[6] J. Thukral and H. Bolcskei, "Interference alignment with limited feedback," in Proc. IEEE. ISIT., Seoul, Korea, Jun. 2009.

[7] R. T. Krishnamachari and M. K. Varanasi, "Interfernce alignment under limited feedback for MIMO interference channels," in Proc. IEEE. ISIT. Austin, Texas, U.S.A, Jun. 2010.

[8] Lingyang Song, Zhu Han, Zhongshan Zhang, and Bingli Jiao, "Noncooperative Feedback Rate Control Game for Channel State Information in Wireless Networks," IEEE Journal on Selected Areas in Communications. special issue on Game Theory in Wireless Communications, vol 30, no. 1, pp. 188-197, 2012.

[9] Lingyang Song, Hong Guo, Bingli Jiao, and Mrouane Debbah, "Joint Relay Selection and Network Coding using Differential Modulation over Two-way Relay Channels," IEEE Transactions on Vehicle Technology, vol. 59, no. 6, pp. 2932 C 2939, Jul. 2010

[10] D. Zhang, G. Wei, J. Zhu and Z. Tian, "On the bounds of feedback rates for pilot-assisted MIMO systems," IEEE. Transactions on Vehicular Thechnology, vol. 56, no. 4, pp. 1727-1736, Jul. 2007.

[11] W. Li, M. Ma, and B. L. Jiao, "Comments 'on the bounds of the feedback rates for pilot-assisted MIMO Systems'," IEEE. Transactions on Vehicular Technology, vol.58, no.8, pp. 4657-4660, Oct. 2009.

[12] K. E. Baddour, and N. C. Beaulieu, "Autoregressive modeling for fading channel simulation" IEEE. Transaction on Wireless Communication, Vol 4, pp. 1650-1662. Jul. 2005. 